\title{
AVALIAÇÃO CLÍNICA E RADIOGRÁFICA DA PREVALÊNCIA DE RESTAURAÇÕES PROXIMAIS COM EXCESSO CERVICAL E CONDIÇÕES PERIODONTAIS ADJACENTES
}

\author{
CLINICAL AND RADIOGRAPHIC EVALUATION OF INTERPROXIMAL \\ OVERHANGING DENTAL RESTORATIONS AND PERIODONTAL STATUS
}

\author{
Claudinéia Wendler Tozetto ${ }^{1}$, Gustavo Pompermaier Garlet ${ }^{2}$, \\ Fábio André dos Santos ${ }^{3}$, Gibson Luiz Pilatti ${ }^{3 *}$ \\ 1 Universidade Estadual de Ponta Grossa - UEPG, PIBIC/CNPq/UEPG \\ 2 Faculdade de Odontologia de Bauru - FOB/USP, Departamento de Ciências \\ Biológicas, Bauru, SP \\ 3* Autor para contato: Universidade Estadual de Ponta Grossa - UEPG, Departamento de \\ Odontologia, Ponta Grossa, PR, Brasil; (42) 3225-0326; e-mail: \\ gibson@tudoparana.com
}

Recebido para a publicação em 21/09/2005

Aceito para a publicação em 04/10/2005

\section{RESUMO}

As restaurações proximais com excesso cervical (REC) representam um importante fator de risco local para perda de inserção periodontal. O objetivo deste estudo foi avaliar a prevalência deste fator etiológico local em 355 indivíduos de ambos os sexos através dos exames clínico e radiográfico, bem como estabelecer a concordância existente entre estes exames. Todas as superfícies proximais dos dentes posteriores presentes na cavidade bucal foram submetidas a um exame clínico, tendo sido classificadas como não restaurada(NR), restaurada sem excesso cervical (RS) e restaurada com excesso cervical (RC). A seguir, estas mesmas superfícies foram examinadas quanto às condições periodontais, aplicando-se os escores utilizados no índice epidemiológico CPITN. O exame radiográfico consistiu na tomada de radiografias interproximais dos dentes posteriores, as quais, após serem processadas, foram avaliadas em negatoscópio com o emprego de uma lupa, a fim de verificar a presença de restaurações proximais RS ou RC, bem como a distância entre a junção esmalte-cemento e a crista óssea alveolar (JCE-CO). Os resultados demonstraram que $44,4 \%$ e $38,8 \%$ das superfícies proximais restauradas exibiram excesso cervical aos exames clínico e radiográfico, respectivamente. Uma maior prevalência de sítios com profundidade de sondagem $>3 \mathrm{~mm}(55,2 \%)$ e com JCE-CO $>2 \mathrm{~mm}$ (77,6\%) foi encontrada em superfícies RC, em comparação às superfícies RS (23\% e 31,8\%, respectivamente). A concordância entre os exames foi boa (valor calculado de Kappa = 0,734). Desta forma, a presença de RECs representa um importante 
fator de risco para a presença de bolsas periodontais e para perda de inserção.

Palavras-chave: restaurações com excesso cervical, prevalência, exame clínico, exame radiográfico, perda de inserção, bolsa periodontal

\begin{abstract}
Overhanging dental restorations (ODRs) are important risk factors for periodontal attachment loss. The aim of this study was to clinically and radiographycally access the prevalence of ODRs in 355 individuals, as well as to establish the agreement between these exams. All the interproximal surfaces of the posterior teeth were examined and classified as not restored (NR), restored without overhangs (RS) and restored with overhangs (RC). The periodontal condition was evaluated using the CPITN scores. Bite wing $\mathrm{x}$-rays were taken, processed and examined to verify the presence of proximal overhanging restorations, as well as the distance from the cementum-enamel junction to the interproximal bone crest (CEJ-BC). The results demonstrated that $44,4 \%$ and $38,8 \%$ of the surfaces with proximal restorations exhibited cervical overhangs on the clinical and radiographic exams, respectively. A higher prevalence of sites with probing depth $>3 \mathrm{~mm}(55,2 \%)$ and with CEJ-BC $>2 \mathrm{~mm}(77,6 \%)$ was found in surfaces RC, in comparison to the RS surfaces (23\% and 31,8\%, respectively). The agreement between the clinical and radiographic examination was considered good, according to the Kappa test $(\mathrm{k}=0,734)$. Thus, the presence of proximal ODRs represents an important risk factor for the presence of periodontal pocket and radiographic attachment loss.
\end{abstract}

Key words: overhanging dental restoration, prevalence, clinical examination, radiographs, periodontal attachment loss, periodontal pocket

\section{Introdução}

Os microrganismos que compõem o biofilme dental representam um dos mais importantes fatores etiológicos da doença periodontal. Outros fatores locais como cálculo dental, apinhamento dentário, presença de dispositivos ortodônticos, restaurações e próteses com adaptação cervical deficiente podem atuar favorecendo a maturação deste biofilme, aumentando a proporção de espécies bacterianas relacionadas à doença periodontal na região do sulco gengival.

Dentre estes fatores locais pré-disponentes, as restaurações proximais com excesso cervical (RECs) estão freqüentemente presentes, representando um importante fator de risco local para perda de inserção periodontal ao longo do tempo. A detecção dos excessos cervicais pode ser feita através do exame clínico, bem como através de exameradiográfico, principalmente pelas técnicas periapical e interproximal.

Em uma revisão de literatura Brunsvold e Lane (1990) verificaram que a prevalência de restauração dental com excesso proximal é muito alta. Pelo menos $25 \%$ das superfícies dentais restauradas apresentavam excesso cervical, e que, portanto, instrumentos táteis como o explorador deveria ser usado em conjunto com radiografias para detectar o maior número de excessos. Maior perda óssea, perda de inserção e inflamação ocorre adjacente a restaurações dentais com excesso cervical comparadas a dentes sem restaurações dentais com excesso, e estes excessos promovem não somente 
acúmulo de placa como também aumenta o número de patógenos periodontais específicos na placa. Ainda segundo os autores, a raspagem e alisamento radicular combinados com instrução de higiene oral são mais efetivos na redução de inflamação gengival quando associados à remoção das restaurações dentais com excesso de material restaurador.

No mesmo ano, Pack, Coxhead e McDonald analisaram 2117 superfícies dentárias restauradas de 100 pacientes, com o objetivo de determinar a prevalência de excessos cervicais e associar com as condições periodontais adjacentes. O exame radiográfico interproximal revelou que $62 \%$ das superfícies proximais examinadas apresentavam excesso cervical. Em cerca de $64 \%$ dos sítios adjacentes a estas restaurações, a profundidade de sondagem era superior a $3 \mathrm{~mm}$, bastante superior em comparação com sítios adjacentes a superfícies não restaurados (23,1\%), ou sítios adjacentes a restaurações sem excesso cervical (49,2\%). A maior prevalência de bolsas com sangramento (32\%) também encontrava-se adjacentes a restaurações com excesso cervical.

Ramalho, Santana e Campos (1994) encontraram uma prevalência de 28,5\% de excessos proximais em restaurações de classe II em amálgama, ao examinarem clinica eradiograficamente (radiografias bite-wing) 57 pacientes.

O objetivo deste estudo foi estabelecer a concordância existente entre o exame clínico e o exame radiográfico para a detecção dos excessos cervicais de restaurações proximais, bem como avaliar a prevalência deste fator etiológico local em indivíduos policiais militares desta comunidade, em comparação aos demais estudos existentes na literatura.

\section{Material e Métodos}

Tomaram parte deste estudo 355 indivíduos de ambos os sexos (25mulheres e 330 homens), com idade entre 21 e 51 anos (média de 33,7 anos), policiais militares membros do Batalhão da Polícia Militar da cidade de Ponta Grossa - PR. Os indivíduos foram primeiramente informados sobre a natureza do estudo, autorizando sua participação voluntária por meio de um termo de consentimento pós-informação. Os pacientes que participassem do estudo poderiam deixar voluntariamente a pesquisa a qualquer momento, necessitando apenas manifestar sua intenção. Foram adotados como critérios de exclusão: pacientes gestantes e pacientes de risco à endocardite bacteriana, que necessitassem de profilaxia antibiótica para a execução da sondagem periodontal.

Inicialmente, todas as superfícies proximais dos dentes posteriores presentes na cavidade bucal foram submetidas a um exame clínico, utilizando-se uma sonda exploradora, a fim de classificar cada superfície como não restaurada (NR), restaurada sem excesso cervical (RS) e restaurada com excesso cervical (RC). A seguir, estas mesmas superfícies foram examinadas quanto às condições periodontais, aplicando-se os escores utilizados no índice epidemiológico CPITN (Índice das Necessidades de Tratamento Periodontal Comunitário), empregando-se para isso uma sonda periodontal preconizada pela Organização Mundial da Saúde (OMS621). Cada superfície proximal foi examinada, atribuindo-se um dos escores do CPITN, de acordo com a condição encontrada: 0-ausência de sangramento; 1presença de sangramento; 2-presença de fatores retentivos locais; 3 - profundidade de sondagem entre 4 e $6 \mathrm{~mm}$; 4- profundidade de sondagem superior a $6 \mathrm{~mm}$.

O exame radiográfico consistiu na realização de tomadas radiográficas interproximais dos dentes posteriores, sendo que todas as medidas de proteção contra as radiações ionizantes preconizadas na literatura foras utilizadas, tais como uso de colete plumbífero e protetor de tireóide, bem como correto emprego da técnica radiográfica e do tempo de exposição (Aparelho Spectro - Dabi Atlante, $60 \mathrm{KW}$, tempo de exposição de 0,3 segundos). As radiografias foram processadas pelo método visual e posteriormente avaliadas em negatoscópio, com o emprego de uma lupa, a fim de verificar a presença de restaurações nas faces proximais, se havia ou não excesso cervical, bem como para determinar, com o emprego de um compasso de ponta seca, a distância entre a junção esmalte-cemento e a crista óssea alveolar (JCE-CO), em milímetros.

Os dados coletados e anotados em fichas próprias foram organizados em tabelas e submetidos à análise estatística para comparação das condições periodontais encontradas em superfícies proximais NR, RS e RC, bem como para determinar o grau de concordância entre os métodos clínico e radiográfico para 
detecção de excessos cervicais de restaurações proximais, através do teste de Kappa.

\section{Resultados}

Foram examinadas um total de 8047 superfícies proximais ao exame clínico, podendo-se constatar que 81,7\% destas superfícies encontravam-se não restauradas, $10,16 \%$ restauradas sem excesso cervical e $8,14 \%$ restauradas com excesso cervical. Os valores das freqüências absolutas encontradas estão expressos na figura 1. Das superfícies restauradas ao exame clínico observou-se que $44 \%$ apresentavam-se com excesso cervical e 56\% encontravam-se sem excesso cervical.

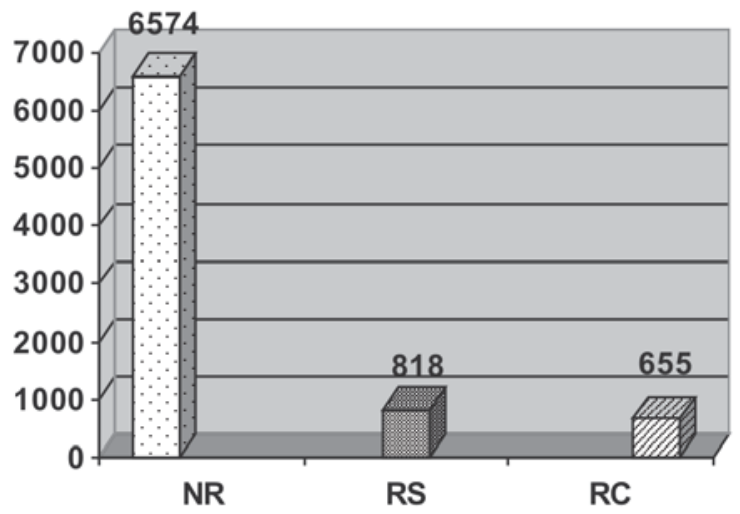

Figura 1 - Freqüências absolutas das superfícies proximais não restauradas (NR), restauradas sem excesso cervical (RS) e restauradas com excesso cervical (RC) ao exame clínico (n=8047).

Na tabela 1 é possível observar as freqüências absoluta e relativa de cada um dos escores do CPITN, de acordo com a condição das superfícies proximais examinadas. Observa-se que quando a superfície encontra-se não restaurada, predominam sítios saudáveis (código 0); quando a superfície passa a ser restaurada há maior prevalência de sítios com sangramento gengival (código 1), ao passo que na presença de excesso cervical, a condição periodontal predominante é a presença de bolsas periodontais entre 4 e 6 mm (código 3).

Tabela 1 - Freqüências absoluta e relativa dos escores do CPITN observados, de acordo com a condição das superfícies proximais examinadas.

\begin{tabular}{lllllll}
\hline & \multicolumn{1}{c}{$\mathbf{0}$} & \multicolumn{1}{c}{$\mathbf{1}$} & \multicolumn{1}{c}{$\mathbf{2}$} & \multicolumn{1}{c}{$\mathbf{3}$} & \multicolumn{1}{c}{$\mathbf{4}$} & Total \\
\hline NR & $2572 / 39,1 \%$ & $2071 / 31,5 \%$ & $825 / 12,5 \%$ & $965 / 14,6 \%$ & $141 / 2,1 \%$ & 6574 \\
RS & $156 / 19 \%$ & $412 / 50,3 \%$ & $62 / 7,5 \%$ & $169 / 20,6 \%$ & $19 / 2,3 \%$ & 818 \\
RC & - & - & $294 / 44,8 \%$ & $338 / 51,6 \%$ & $23 / 3,5 \%$ & 655 \\
\hline Total & 2728 & 2483 & 1181 & 1472 & 183 & 8047 \\
\hline
\end{tabular}

*teste de Qui-quadrado significante ao nível de 5\%

As freqüências absoluta e relativa dos sítios que apresentaram profundidade clínica de sondagem $\leq 3 \mathrm{~mm}$ (escores 0, 1 e 2 do CPITN) e dos sítios que apresentaram profundidade clínica de sondagem $>3 \mathrm{~mm}$ (escores 3 e 4 do CPITN) estão expressos na tabela
2, onde observamos que enquanto nas superfícies NR e RS há maior frequência de sítios com profundidade de sondagem $\leq 3 \mathrm{~mm}$, nas superficies RC a frequência maior é de sítios com profundidade de sondagem $>3 \mathrm{~mm}$. 
Tabela 2 - Freqüências absoluta e relativa dos sítios que apresentaram profundidade clínica de sondagem $\leq 3 \mathrm{~mm}$ (escores 0 , 1 e 2 do CPITN) e dos sítios que apresentaram profundidade clínica de sondagem > 3mm (escores 3 e 4 do CPITN).

\begin{tabular}{llll}
\hline $\begin{array}{l}\text { Condição da } \\
\text { superfície analisada }\end{array}$ & PS $\leq \mathbf{3 m m}$ & PS>3mm & Total \\
\hline NR & $5468 / 83,1 \%$ & $1106 / 16,8 \%$ & 6574 \\
RS & $630 / 77 \%$ & $188 / 23 \%$ & 818 \\
RC & $294 / 44,8 \%$ & $361 / 55,2 \%$ & 655 \\
\hline Total & 6392 & 1655 & 8047 \\
\hline
\end{tabular}

*teste de Qui-quadrado significante ao nível de 5\%

Frente ao exame radiográfico foi possível avaliar 6685 superfícies, onde encontramos 79,2\% das superfícies classificadas como NR, 12,8\% classificadas como RS e 8,0\% classificadas como RC. As frequên- cias absolutas estão expressas na figura 2. Do total de superfícies restauradas, $61,2 \%$ encontravam-se sem excesso cervical e 38,8\% apresentavam-se com excesso cervical.

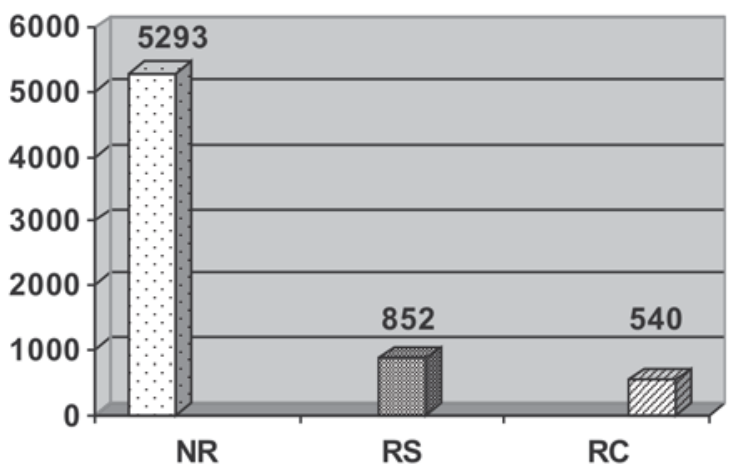

Figura 2 - Freqüências absolutas das superfícies proximais não restauradas (NR), restauradas sem excesso cervical (RS) e restauradas com excesso cervical (RC) ao exame radiográfico ( $n=6685)$.

Na tabela 3 observa-se as freqüências absoluta e relativa dos sítios que apresentaram a distância da junção amelocementária à crista óssea (JCE-CO) $\leq 2 \mathrm{~mm}$ ou distância JCE-CO $>2 \mathrm{~mm}$ frente ao exame radiográfico para as diferentes condições de superfícies proximais avaliadas, onde podemos verificar que superfícies RC apresentam com maior freqüência a distância da junção amelocementária à crista óssea $>2 \mathrm{~mm}$ em comparação à superfícies NR e RS.

Tabela 3 - Freqüências absoluta e relativa dos sítios que apresentaram a distância da junção amelocementária à crista óssea $(\mathrm{JCE}-\mathrm{CO}) \leq 2 \mathrm{~mm}$ ou distância JCE-CO $>2 \mathrm{~mm}$ frente ao exame radiográfico $(\mathrm{n}=6685)$.

\begin{tabular}{llll}
\hline & JCE-CO $\leq \mathbf{2 ~ m m}$ & JCE-CO $>\mathbf{2 m m}$ & Total \\
\hline NR & $3856 / 72,8 \%$ & $1437 / 27,2 \%$ & 5293 \\
RS & $581 / 68,2 \%$ & $271 / 31,8 \%$ & 852 \\
RC & $121 / 22,4 \%$ & $419 / 77,6 \%$ & 540 \\
\hline Total & 4558 & 2127 & 6685 \\
\hline
\end{tabular}

*teste Qui-quadrado significante ao nível de 5\%. 
A concordância entre os exames clínico e radiográfico para a detecção de superfícies com ou sem ex-cesso cervical de restauração foi determinada pelo teste de Kappa, constatando-se uma concordância entre os exames em 86,9\% dos casos, obtendo-se um valor de $\mathrm{K}=0,734$, considerada uma boa concordância, como observado na tabela 4.

Tabela 4 - Concordância entre os exames clínico e radiográfico para a detecção de superfícies com ou sem excesso cervical de restauração, determinada pelo teste de Kappa.

\begin{tabular}{llll}
\hline Exame Radiográfico & \multicolumn{2}{c}{ Exame Clínico } \\
\cline { 2 - 4 } & RS & RC & Total \\
\hline RS & 720 & 143 & 863 \\
RC & 43 & 515 & 558 \\
\hline Total & 763 & 658 & 1421 \\
\hline
\end{tabular}

\section{Discussão}

Diversos estudos vêm sendo realizados ao longo dos anos com o propósito de investigar a prevalência de RECs e sua possível influência sobre as condições dos tecidos periodontais adjacentes, como pode ser visto no quadro abaixo:

Quadro 1 - Síntese dos estudos realizados para determinar a prevalência de RECs.

\begin{tabular}{|c|c|c|c|c|}
\hline Autores & $\begin{array}{l}\text { Método de } \\
\text { Exame }\end{array}$ & $\begin{array}{c}\mathrm{N}^{0} \text { de } \\
\text { Pacientes }\end{array}$ & $\begin{array}{c}\mathrm{N}^{0} \text { de Dentes ou } \\
\text { Superfícies Estudados }\end{array}$ & $\begin{array}{c}\text { Prevalência de } \\
\text { Restaurações com } \\
\text { Excesso }\end{array}$ \\
\hline $\begin{array}{c}\text { Gilmore e Scheihan } \\
\text { (1971) }\end{array}$ & $\begin{array}{l}\text { Exame clínico e } \\
\text { radiográfico }\end{array}$ & 1976 & - & $\begin{array}{l}25 \% \text { das superfícies } \\
\text { restauradas } \\
32 \% \text { dos indivíduos }\end{array}$ \\
\hline Burch et al. (1976) & $\begin{array}{l}\text { Exame clínico e } \\
\text { radiográfico }\end{array}$ & 852 & 16365 superfícies & $\begin{array}{l}30 \% \text { das superfícies } \\
\text { restauradas }\end{array}$ \\
\hline $\begin{array}{l}\text { Coxhead, Robertson } \\
\text { e Simpson (1978) }\end{array}$ & $\begin{array}{c}\text { Exame } \\
\text { radiográfico }\end{array}$ & 184 & 2108 superfícies & $\begin{array}{l}52 \% \text { das superfícies } \\
\text { restauradas }\end{array}$ \\
\hline $\begin{array}{c}\text { Than, Duguid e } \\
\text { McKendrick (1982) }\end{array}$ & Exame clínico & - & 240 dentes extraídos & $\begin{array}{c}60 \% \text { das superfícies } \\
\text { restauradas }\end{array}$ \\
\hline Claman et al. (1986) & $\begin{array}{c}\text { Exame } \\
\text { radiográfico }\end{array}$ & 826 & - & $\begin{array}{l}27,2 \% \text { das superfícies } \\
\text { restauradas }\end{array}$ \\
\hline Franco et al. (1987) & $\begin{array}{c}\text { Exame } \\
\text { radiográfico }\end{array}$ & 204 & - & $\begin{array}{c}42,2 \% \text { das superficies } \\
\text { restauradas }\end{array}$ \\
\hline $\begin{array}{l}\text { Pack, Coxhead e } \\
\text { McDonald (1990) }\end{array}$ & $\begin{array}{l}\text { Exame clínico e } \\
\text { radiográfico }\end{array}$ & 100 & 1319 dentes & $\begin{array}{c}56 \% \text { das superfícies } \\
\text { restauradas }\end{array}$ \\
\hline $\begin{array}{l}\text { Ramalho, Santana e } \\
\text { Campos (1994) }\end{array}$ & $\begin{array}{l}\text { Exame clínico e } \\
\text { radiográfico }\end{array}$ & 57 & 1510 superficies & $\begin{array}{l}29,5 \% \text { das superfícies } \\
\text { restauradas }\end{array}$ \\
\hline
\end{tabular}

Reiterando os estudos anteriores, este estudo constatou que atualmente as RECs continuam apresentando uma alta prevalência (38 a 44\%), demonstrando que mesmo conhecendo o problema, nenhum cuidado adicional tem sido adotado para diminuir esta prevalência. 
Foi possível observar ao exame clínico um aumento na prevalência de sítios com profundidade clínica de sondagem maior que $3 \mathrm{~mm}$ (escore 3 do CPITN), tanto em superfícies restauradas com ou sem excesso cervical. Ao exame radiográfico observa-se que a freqüência de sítios com a distância JCE-CO $\leq 2 \mathrm{~mm}$ diminui na presença de superfícies proximais restauradas. De grande relevância é a alta freqüência de sítios com reabsorção da crista óssea interproximal quando da presença de REC.

Na literatura há estudos que discutem se a condição das faces proximais tem alguma influência sobre as condições do periodonto adjacente. Keszthelyi, Szabo e Strahan (1985), afirmam que lesões cariosas classe II amplas e abertas não são consideradas como um fator importante na perda de inserção. Pinto et al. (2000) e Sant'Ana e Passanezi (2002) verificaram em seus estudos que a presença de restauração também não é responsável por uma maior perda de inserção periodontal quando comparado a dentes não restaurados. Porém, este estudo concorda com Keszthelyi e Szabo (1984) e Chen et al. (1987) ao afirmarem que uma face restaurada tende a apresentar maior perda de inserção quando comparada a outra face não restaurada.

Quando a face restaurada apresenta excesso cervical, Arneberg, Silness e Nordbo (1980) e Than, Duguid e McKendrick (1982) defendem a idéia de que não existe correlação entre a severidade da doença gengival e a presença de restauração com excesso cervical. Por outro lado, a maioria dos estudos encontrados na literatura encontraram maior destruição dos tecidos periodontais adjacentes à RECs, detectadas por meio de exame clínico e/ou radiográfico (Trott e Sherkat, 1964; Gilmore e Sheiham, 1971; Highfield e Powell, 1978; Gorzo, Newman e Strahan, 1979; Rodriguez-Ferrer, Strahan e Newman, 1980; Lang, Kiel e Anderhalden,1983; Claman, Koidis e Burch,1986; Coxhead,1986; Eid,1987; Brunsvold e Lane,1990; Pack, Coxhead e McDonald,1990; Jansson et al.,1994; Nogueira-Filho et al., 2001; e Matthews e Tabesh, 2004).

Os achados deste estudo corroboram com diversos autores no que diz respeito à maior perda óssea interproximal próximo à RECs, em relação às faces não restauradas ou restauradas sem excesso cervical
(Gilmore e Sheiham,1971; Burch, Garrity e Schnecker,1976; Jeffcoat e Howell, 1980; Hakkarainen e Ainamo, 1980; Keszthelyi e Szabo, 1984; Claman, Koidis e Burch, 1986; Eid,1987; Brunsvold e Lane, 1990; Pack, Coxhead e Mcdonald, 1990; Jansson et al., 1994; Parsell et al., 1998).

As RECs, apesar de apresentarem uma alta prevalência, não são comumente detectadas por muitos profissionais, pois a associação de exames clínico e radiográfico não é uma pratica rotineira, e mesmo tendo encontrado uma boa concordância entre estes dois exames (86,91\%), eles devem ser usados em conjunto para uma maior segurança quanto ao diagnóstico.

Quanto à metodologia utilizada para a detecção de RECs, cabe ressaltar que o emprego de radiografias interproximais como exame complementar é efetivo na determinação de alterações na crista óssea interproximal e sua relação com a junção amelo-cementária e com restaurações proximais (Kelly, 1975; Schüller e Holst, 1996; Lang e Hill, 1977; Gorzo, Newman e Strahan, 1979; Arneberg, Silness e Nordbo, 1980; e Pack, 1989).

Em um outro estudo, Jenkins, Cross e Kinane (1994) constataram que, quando há restauração com excesso cervical, este pode também estar associado à presença de uma fenda marginal, e que quanto maior o excesso de material, maior tende a ser esta fenda. Este estudo sugere que a prática de recontorno dos excessos, com a finalidade de promover saúde periodontal, poderá ser ineficaz, pois a retenção de placa provavelmente continuará presente dentro da fenda marginal.

Por fim, é importante a correta execução das técnicas restauradoras para evitar a ocorrência de excessos cervicais, os quais têm um efeito deletério sobre o periodonto. Além disso, deve-se utilizar, sempre que necessário, uma associação dos métodos clínico e radiográfico para a detecção de RECs no exame e diagnóstico dos pacientes com doença periodontal, visto que representam um importante fator de rico para a perda de inserção e progressão da doença periodontal.

\section{Conclusões}

Pode-se concluir com base nos resultados ob- 
tidos que: 1 - Há uma alta incidência de REC na amostra estudada (44\% e 39\% aos exames clínico e radiografico, respectivamente); 2 - Os sítios periodontais adjacentes à RC apresentam maior incidência de bolsas periodontais e reabsorção da crista óssea alveolar interproximal do que sítios NR e RS; desta forma, a presença de superfícies proximais RC representa um importante fator de risco para a presença de bolsas periodontais e para perda de inserção. 3 - A concordância entre os exames clínico e radiográfico quando da detecção de REC (valor de Kappa = 0,734) é considerada boa.

\section{REFERENCIAS}

1. ARNEBERG P.; SILNESS, J.; NORDBO, H. Marginal fit and cervical extent of class II amalgam restorations related to periodontal condition. Journal of Periodontal Research 15: 669-677, 1980 .

2. BRUNSVOLD, M. A.; LANE, J. J. The prevalence of overhanging dental restorations and their relationship to periodontal disease. J Clin Periodontol 17: 67-72, 1990.

3. BURCH, J. G.; GARRITY, T.; SCHNECKER, D. Periodontal Pocket Depths Related to Adjacent Proximal Tooth Surface Conditions and Restorations. Journal of the Kentucky Dental Association 13-18, oct.1976.

4. CHEN, J. J. et al. Periodontal attachment loss associated with proximal tooth retorations. The Journal of Prosthetic Dentistry 57:4: 416-420, April 1987.

5. CLAMAN, L. J.; KOIDIS, P. T.; BURCH, J. G. Proximal tooth surface quality and periodontal probing depth. JADA 113: 890-893, dec. 1986.

6. COXHEAD, L. J.; ROBERTSON, J. B.; SIMPSON, E. F. Amalgam overhangs - a radiographic study. New Zeland Dental Jornal 74: 145-147, 1978.

7. COXHEAD, L. J. Amalgam overhangs: a major cause of periodontal disease. New Zeland Dental Jornal 82: 99-101, 1986.

8. FRANCO, E. B. et al. Avaliação radiográfica de restaurações a amalgama com excessos proximais. Rev. Odont. USP, 1(2):1619, abr./jun. 1987.

9. GILMORE, N.; SHEIHAM, A. Overhanging Dental Restorations and Periodontal Disease. J. Periodontol. 42:1: 812, jan. 1971.

10. GORZO, I.; NEWMAN, H. N.; STRAHAN, J. D. Amalgam restorations, plaque removal and periodontal health. J Clin Periodontol 6: 98-105, 1979.

11. HAKKARAINEN, K.; AINAMO, J. Influence of overhanging posterior tooth restorations on alveolar boné height in adults. J Clin Periodontol 7: 114-120, 1980.

12. HIGHFIELD, J. E.; POWELL, R. N. Effects of removal of posterior overhanging metallic margins of restorations upon the periodontal tissues. J Clin Periodontol 5: 169-181, 1978.

13. JANSSON, L. et al. Proximal restorations and periodontal status. J Clin Periodontol 21: 577-582, 1994.

14. JEFFCOAT, M. K.; HOWELL, T. H. Alveolar Bone Destruction Due to Overhanging Amalgam in Periodontal Disease. J. Periodontol. 51:10: 599-602, oct. 1980.

15. JENKINS, W. M. M.; CROSS, L. J.; KINANE, D. F. Cervical marginal fit of proximal amalgam restorations. J. Dent. 22: 108111, 1994.

16. KELLY, G. P. et al. Radiographs in Clinical Periodontal Trials. J. Periodontol. 46:7: 381-386, jul., 1975.

17. KESZTHELYI, G;; SZABO, I. Influence of Class II amalgam filings on attachment loss. J Clin Periodontol 11: 81-86, 1984.

18. KESZTHELYI, G.; SZABO, I.; STRAHAN, J. D. Loss of attachment adjacent to class II carious lesions. J Clin Periodontol 12: 405-410, 1985

19. LANG, N. P.; HILL, R. W. Radiographs in periodontics. J Clin Periodontol 4: 16-28, 1977.

20. LANG, N. P.; KIEL, R. A.; ANDERHALDEN, K. Clinical and microbiological effects of subgingival restorations with overhanging or clinically perfect margins. J Clin Periodontol 10: 563-578, 1983.

21. M. EID, B. D. S. Relationship between overhanging amalgam restorations and periodontal disease. Quintessence International 18:11: 775-781, 1987.

22. MATTHEWS, D. C.; TABESH, M. Detection of localized tooth-related factors that predispose to periodontal infections. Periodontology 2000 34: 136-150, 2004.

23. NOGUEIRA-FILHO, et al. Necessidade de tratamento periodontal avaliada pelo CPITN e sua relação com a qualidade de acabamento cervical das restaurações. Pesqui Odontol Bras 15:1: 51-55, jan./mar. 2001.

24. PACK, A. R. C. The amalgam overhang dilema: a review of causes and effects, prevention, and removal. New Zeland Dental Jornal 85: 55-58, 1989.

25. PACK, A. R. C.; COXHEAD, L. J.; McDONALD, B. W. The prevalence of overhang margins in posterior amalgam restorations and periodontal cansequences. J Clin Periodontol 17: 145-152, 1990.

26. PARSELL, D. E. et al. The effect of amalgam overhangs on alveolar bone height as a function of patient age and overhang width. Operative Dentistry 23: 94-99, 1998.

27. PINTO, F. R. et al. Inluência de Restaurações em amálgama classe II no nível de inserção periodontal. Periodontia Revista 
9:1: 19-24, jan./jun. 2000.

28. RAMALHO, L. M. P.; SANTANA, E. J. B.; CAMPOS, P. S. F. Estudo da prevalência de restaurações de amálgama com excessos proximais. Rev. paul. Odontol. 16(5): 28-32, set./out. 1994.

29. RODRIGUEZ-FERRER, H. J.; STRAHAN, J. D.; NEWMAN, H. N. Effect on gingival health of removing overhanging margins of interproximal subgingival amalgam restorations. $\mathbf{J}$ Clin Periodontol 7: 457-462, 1980.

30. SANT'ANA, A. C. P.; PASSANEZI, E. Pattern of periodontal disease progression in restored versus non- restored teeth. Rev Fac Odontol Bauru 10:4: 225-230, 2002.

31. SCHÜLLER, A. A.; HOLST, D. Testing the consistency of measurements of the distance between the cemento-enamel junction and the alveolar bone crest on bitewing radiographs. J Clin Periodontol 23: 977-981, 1996.

32. THAN, A.; DUGUID, R.; McKENDRICK, A. J. W. Relationship between restorations and the level of the periodontal attachment. J Clin Periodontol 9: 193-202, 1982.

33. TROTT, J. R.; SHERKAT, A. Effect of class II amalgam restorations on health of the gingiva: a clinical survey. $\mathbf{J}$. CANAD.D.A. 30:12: 766-770, 1964. 